\title{
Specifics of determining the tension forces of the cable-stayed bridge elements
}

\author{
Ivan Chaplin ${ }^{1, *}$, and Andrey Yashnov ${ }^{1}$ \\ ${ }^{1}$ Siberian Transport University, Dusi Kovalchuk st., 191, 630049, Novosibirsk, Russia
}

\begin{abstract}
Objective: to improve the method of determining the force in the cable stays by frequencies of natural transverse oscillations. Methods: synthesis and analysis of theoretical and experimental data, experimental methods for studying the structures. Results: in the paper, the problems of determining the tension forces of the cable stays by the frequencies of their natural oscillations are considered taking into account various factors (the design of anchor fastenings, the angle of inclination and sagging of the cable stay, the change of temperature). The estimation of possible errors in calculations is given. The degree of coincidence of the actual and design stress-strain state of the cable stays depends on the correctness of accounting the influence of the factors considered. The effect of the temperature change and the anchor fastening structure on the frequency of the natural oscillations of the cable stays has been experimentally tested. The recommendations on the determination of the forces in the cable stays by dynamic parameters are given. A method for controlling the tension forces of the cable stays and specialized software "Vant" ("Cable") for automation of the measurement process are developed. The method has been tested on several bridge structures that have cable-stayed elements. Practical significance: the ability to use the developed method for controlling the tension forces of cable-stayed elements during the operation of artificial structures.
\end{abstract}

\section{Introduction}

Cable stays are the main supporting element in cable-stayed bridges. Typically, to ensure the design distribution of forces in the cable-stayed girder, pre-tension of cable stays is implemented during the installation. If in the course of operation, for some reason, the tension forces change, this leads to a change in the stress-strain state of the structure as a whole. In the absence of proper monitoring of the tension forces in the cable stays, the probability of a sudden achievement of the limiting state by the construction increases.

The experience of monitoring unique cable-stayed and suspended foreign bridges is reflected in the study [1], which noted that the measurement of forces in cable-stayed elements can be carried out using accelerometers and magnetoelastic sensors. Also, generalized theoretical and experimental studies on the design and construction of cablestayed bridges, in particular, the features of static and dynamic operation of cable-stayed

\footnotetext{
* Corresponding author: ivannumber1_chaplin@mail.ru
} 
elements; recommendations on accounting the stress-strain state of the cable stays in the areas of attachment to the anchors, and other information relating to cable-stayed bridges are collected in [2].

Large-scale domestic studies of the dynamic work of the cable-stayed system were carried out during the design, construction, and the first years of operation of the Ugra Bridge across the river Ob near the city of Surgut [ $3 \ldots$ 7]. It should be noted that the Ugra Bridge is the longest single-pylon cable-stayed bridge with the length of the overlapped span - $408 \mathrm{~m}$ [3], for this reason, leading specialized organizations of Russia were involved in its construction and design.

The thesis of A.P. Sychev [5], performed on the basis of the Research Institute of Transport Construction (CNIIS), presents the results of a study of methods for estimating the stress-strain state of the cable stays, describes the features of constructing the finite element model of the Ugra Bridge. Of special interest are the graphs [5, p.82], which clearly demonstrate the change in the stresses in the cable-stayed elements (on the example of the Ugra Bridge) in the anchor zone, which act as stretched rods with a bend, which was also noted in the Recommendations [2].

The work of S.N. Dyadkin [4] presents the results of the monitoring of cable-stayed span structures taking into account climatic factors on the example of a bridge across the river $\mathrm{Ob}$ near the city of Surgut. The experience of using various methods of determining the tension forces of the cable stays during the construction of the Ugra Bridge (by the sagging of the cable stays, by the frequencies of natural oscillations, by the change in local deformations recorded in the anchors, and by the readings of the pressure gauges on jacks) is described. It is noted that the tension forces of the cable stays determined from the change in local deformations recorded in the anchors, in some cases, were overestimated in comparison with the values of the forces determined from the readings of the pressure gauges on jacks.

Specialists of Zhukovsky TsAGI carried out complex studies of the aerodynamic stability of the pylon and the span structure of the Ugra Bridge, including a large-scale model in a wind tunnel [3, 4]. Specialists of Zhukovsky TsAGI [7] measured the forces in the cable stays in the first four years of the bridge operation using a specially developed technique described in detail in $[3,6,7]$.

The thesis of M.I. Khazanov [8] analyzed the method of determining the force in the cable stay by the velocity of the wave of oscillations. The velocity of the wave of oscillations is measured by installing two longitudinal piezoelectric sensors. The accuracy of determination of the forces in the cable stay increases with the increase in the report rate and the distance between the piezoelectric sensors. It is quite difficult to install two sensors on a cable stay with a large distance between each other, and the length of the cable stays themselves is often insufficient to ensure a measurement accuracy of $5-10 \%$, so the use of this method is limited.

The problems of estimating the stress-strain state of cable-stayed elements are also reflected in other works $\left[\begin{array}{lll}9 & \ldots & 15\end{array}\right]$. Of course, most of the experimental studies (especially domestic ones) are limited to analyzing the work of one or two structures, which narrows the area of their possible application on other cable-stayed structures. However, in general, theoretical and experimental studies of the work of cable-stayed elements have recently developed. Monitoring of the stress-stain state of cable-stayed systems is carried out on the cable-stayed bridges of Europe, Japan, and China. With the participation of the authors, complex studies of the work of cable-stayed elements in the structure of bridge structures have been carried out. Based on its results, a method for determining the strength of the cable-stayed element was suggested [16]. The essence of the method lies in the improvement of the classical formula for determining the tension force of the cable-stayed 
element with respect to the frequencies of its own transverse oscillations [9, p. 182] by refining the parameters (length and mass) included in this formula.

The purpose of this study is to improve the method for determining the force in the cable stays by frequencies of natural transverse oscillations.

\section{Materials and Methods}

An idealized solution for determining the force of a cable-stayed element by the frequencies of its own transverse oscillations can be obtained by taking into account the classical formula [9]:

$$
\omega_{n}=\frac{n \pi}{l} \sqrt{\frac{N}{m}}
$$

where $\omega_{n}-n$-th circular oscillation frequency of a cable stay, $\mathrm{Hz} ; n$ - the sequence number of the oscillation mode of a cable stay; $l$ - length of a cable stay, m; $N$ - the tension force of a cable stay, $\mathrm{N} ; m-\quad$ mass per unit length of a cable stay, $\mathrm{kg} / \mathrm{m}$.

The dependence of the natural and circular oscillation frequencies is expressed by the following formula:

$$
v_{n}=\frac{\omega_{n}}{2 \pi}
$$

With allowance for (1) and (2), the frequency of the natural transverse oscillations of the cable stay is determined by the formula:

$$
v_{n}=\frac{n}{2 l} \sqrt{\frac{N}{m}}
$$

However, using this simple formula, it is impossible to take into account possible deviations in the actual operation of the structure from an idealized design scheme. Therefore, the authors have analyzed the existing theoretical developments and carried out complex experimental studies of the dynamic work of the cable stays as a part of bridge structures.

The method for determining the tension force of the cable-stayed element [16] was developed on the basis of the results of control of the forces in cable-stayed suspensions of the Bugrinsky Bridge across the river Ob in Novosibirsk in the process of construction and the initial period of operation $[10,15]$. Approbation and verification of the method [16] was carried out in the process of monitoring the forces in the cable stays of the bridge across the river Irtysh on the bypass of Pavlodar [14, 15], Vinogradovsky Bridge across the Tatyshev channel in Krasnoyarsk [17], Ugra Bridge across the river Ob near the city of Surgut [13]. Experimental studies were carried out using a small measuring complex "Tensor MS" developed in the research laboratory "Bridges" of SSTU $[18,19]$. To measure the response of structures to external influences, sensitive vibration sensors-accelerometers (measuring range from $-610 \cdot 10^{-3}$ to $+610 \cdot 10^{-3} \mathrm{~m} / \mathrm{s}^{2}$, sampling frequency $-100 \mathrm{~Hz}$ ) and strain gauges (measuring range from $-1000 \cdot 10^{-9}$ to $+1000 \cdot 10^{-9} \mathrm{~m}$, the inaccuracy of deformation measurements is $\pm 1.5 \%$ ) were used. The vibration records were processed using specialized software "Tensor MS" [19] and "Vant" ("Cable stay") [20], in which the algorithm for fast Fourier transform with Welch window transformation is implemented.

The choice of the range of parameters of cable-stayed elements in the framework of theoretical studies is based on the actual characteristics of the cable-stayed elements of the bridges on which the force measurements were made: length $l=5 \ldots 400 \mathrm{~m}$; tension force $N$ $=100 \ldots 2150 \mathrm{kN}$; number of strands $-8 \ldots 30 \mathrm{pcs}$. (the area of a solid cross-section with a diameter of $72 \mathrm{~mm}$ is comparable to the area of 30 strands); the elastic modulus of the 
metal $E=1.9 \cdot 10^{5} \mathrm{MPa}$; the mass per unit length of a single strand is $1.29 \mathrm{~kg} / \mathrm{m}$; the mass per unit length of the external cable stay covering is $2.32 \mathrm{~kg} / \mathrm{m}$; the angle of inclination to the horizon is $20 \ldots 90^{\circ}$.

The main methods used in the paper are synthesis and analysis of theoretical and experimental data, as well as experimental methods for studying structures.

\section{The results of theoretical studies}

According to known formulas, the effect of various factors (sagging, anchor designs, tilt of the cable stay, bending stiffness of the cable stay, temperature) on the change in the design tension force of the cable stay is calculated. Calculations were made for structures with cable-stayed elements, in the survey of which the authors took part.

\subsection{Effect of sagging on the tension force of the cable stay}

Under the influence of its own weight, the cable stay will deform in shape close to the outline of the parabola $[2,5,11]$. Moreover, on the inclined cable stays, the point of maximum of the parabola will be shifted to the unit of the bottom fixation. However, the effect of this shift is not significant when determining the maximum sag, as noted in [2]. Therefore, the frequency of natural transverse oscillations of the cable stay with allowance for its sagging can be determined by the formula [1]:

$$
v_{n}^{S}=\frac{n}{2 l} \sqrt{\frac{N}{m}+\frac{E A f_{s}^{2}}{2 m l^{2}}}
$$

where $A$ - total cross-sectional area of the cable stay, $\mathrm{m}^{2} ; E$ - modulus of elasticity of the cable stay, $\mathrm{Pa} ; f_{\mathrm{s}}$ - the maximum sagging of the cable stay, $\mathrm{m}$, determined by the formula [2]:

$$
f_{s}=\frac{q l^{2}}{8 N}
$$

where $q=m g-$ linear distributed gravity load of the cable stay, $\mathrm{N} / \mathrm{m}$.

The force in the cable stay, taking into account the influence of its sagging in accordance with the formula (4), can be determined by the formula:

$$
N_{\text {w.s.v. }}=N+N_{s}
$$

where $N_{s}=\frac{E A f_{s}{ }^{2}}{2 l^{2}}-$ force arising from the sagging of the cable stay, $\mathrm{N}$.

To assess the influence of various factors on the force in the cable stay, the relative error $\delta_{f}$ is determined by the following formula:

$$
\delta_{f}=\frac{N_{f}}{N} 100 \%
$$

where $N_{f}=N_{s}$ - the force in the cable stay created by the factor under consideration (in this case, the additional force arising from the sagging), N.

The relative error $\delta_{s}$ of the tension force of the cable-stayed element without taking into account the effect of its sagging depends on four parameters - $N, m, A$, and $l$, two of which: the mass per unit length $m$ and the area of the cable stay $A$ can be replaced by one - the number of strands in the cable stay. Figure 1 shows the surface of $\delta_{s}$. When constructing the surface, the value of the tension force of the cable stay is conventionally taken to be 935 $\mathrm{kN}$ (the average value of all the forces in the cable stays of the operated bridges measured with the participation of the authors). Obviously, the values of the relative errors for other 
forces are inversely proportional to the ratio of the value of the force to the value adopted when constructing the surface.

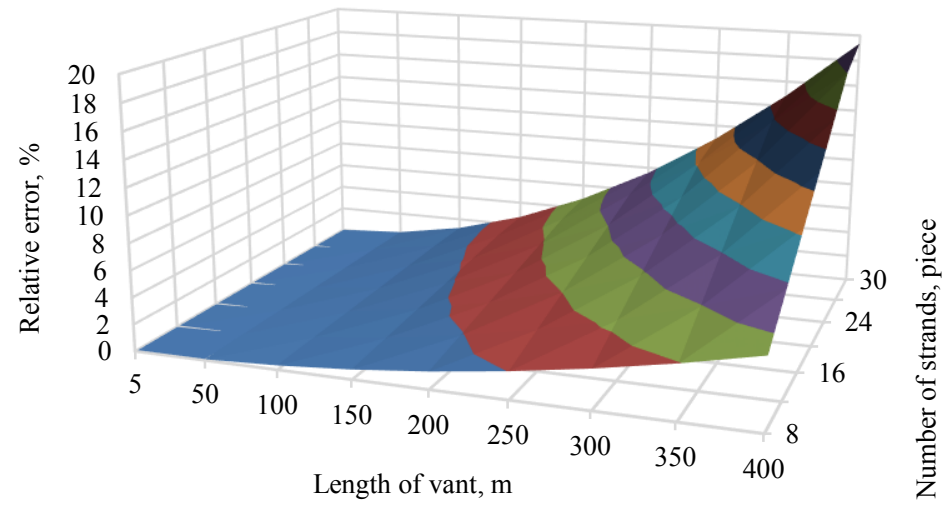

Fig. 1. Surface of the relative error of the tension force of the cable-stayed element without taking into account the effect of its sagging.

As can be seen from the surface in Figure 1, the relative error $\delta_{s}$ increases with the increasing length and cross section of the cable stay as a function of the nonlinear dependence. The relative error of the tensile force of the cable-stayed element without taking into account the effect of its sagging can reach 100 and more \%. However, usually the ratio of the parameters of the cable-stayed elements of the bridges is that the relative error of the tension force of the cable-stayed element without taking into account the effect of its sagging does not exceed 3\%.

\subsection{Influence of the fastening structures on the tension force of the cable stay}

Errors in the determination of forces, taking into account the classical formula (1), also arise from the absence of an ideal hinge in the fastening units of the cable stays. It should be noted that the axis of the anchor must coincide with the tangent drawn to the profile of the cable stay at the point of connection with the anchor. When forming an angle between these lines, the cable stay will work at the fastening point as a stretched element with a bend [5]. Moreover, the bending moment will appear on the front side of the anchor (in the plane of rigid fixation) and decrease according to the exponential dependence $[2,11]$. For example, it is indicated in [5] that additional bending stresses led to the breakage of zshaped wires in several cable stays of the Ugra Bridge (at the junctions of the cable stays with the pylon). If to design a smooth transition of the change in stiffness in the anchor zone, i.e. to make the shape of the oscillations more flat at the place where the cable stay is attached, the value of the bending moment will decrease [11].

However, such structures, as shown by experimental studies, participate in the oscillatory process with the cable stays, which was also noted in [6] in the studies of the cable-stayed system of the Ugra Bridge carried out by specialists of Zhukovsky TsAGI. The analytical solution of the problem of oscillations of the cable stay together with the fastening structures is a complex problem.

Therefore, when switching from a real research object to a design scheme, simplification was introduced through extension of the cable stay by the length of the anchor. Figure 2 shows the profile variants of the first form of the oscillation of the cable stay, taking into account the fastening structures. 
a) without taking into account the inclusion of the bottom anchor structure in the oscillatory process

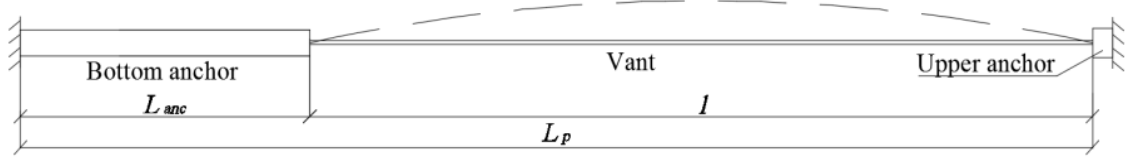

b) the actual oscillation profile of the cable stay together with the bottom anchor structure



c) the accepted oscillation profile of the cable stay together with the anchor

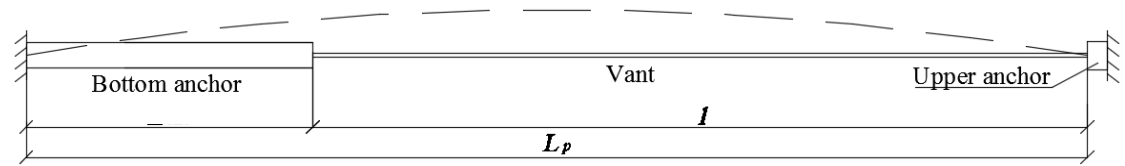

Fig. 2. Profiles of the first form of the cable stay oscillations.

The relative error in determining the tension forces of the cable stays without taking into account the length of the anchor $L_{\text {anc }}$ can be calculated by the formula:

$$
\delta_{l}=\frac{L_{p}^{2}-l^{2}}{l^{2}} 100 \%
$$

where $L_{p}=l+L_{a n c}$ - design length of the cable stay (taking into account the length of the anchor), $\mathrm{m}$.

Figure 3 shows a graph of the relative error in the tension force of the cable stays without taking into account the length of the anchor $\left(L_{a n c}=1.35 \mathrm{~m}-\right.$ the length of the bottom anchors of the Bugrinsky bridge).



Fig. 3. The graph of the relative error in the tension force of the cable stays without taking into account the length of the anchor.

As can be seen from the graph (see figure 3), when determining the tension forces of the cable stays without taking into account the length of the anchor by the frequencies of their natural transverse oscillations, large relative errors (more than 50\%) can arise. Therefore, to eliminate the errors associated with the influence of anchors participating in the joint oscillatory process with the cable stays, it is necessary to take into account the length of the anchors, which is confirmed by numerous experimental studies [13-16, etc.]. 


\subsection{Effect of the angle of inclination on the tension force of the cable stay}

The frequency of the natural transverse oscillations of the cable-stayed element, taking into account the influence of the angle of inclination to the horizon, can be determined by the following formula [12]:

$$
v_{n}^{\alpha}=\frac{n}{2 l} \sqrt{\frac{N}{m}-\frac{g l \sin \alpha}{2}}
$$

where $\alpha$ - angle of inclination to the horizon, degrees;

Taking (9) into account, we obtain:

$$
N_{\text {w.t.a. }}=N-N_{\alpha}
$$

where $N_{\alpha}=\frac{m l g \sin \alpha}{2}-$ force, depending on the angle of inclination of the cable stay to the horizon, $\mathrm{N}$.

A theoretical study of the influence of the angle of inclination to the horizon on the variation in the tension force of the cable stay was carried out by analyzing the surfaces of the relative error $\delta_{\alpha}$, constructed analogously to the surface in Figure 1 by the formula (7). For example, in Figure 4, the surface of the relative error $\delta_{\alpha}$ is plotted with the tensile force of a cable stay made of 12 strands, which is taken to be $935 \mathrm{kN}$.
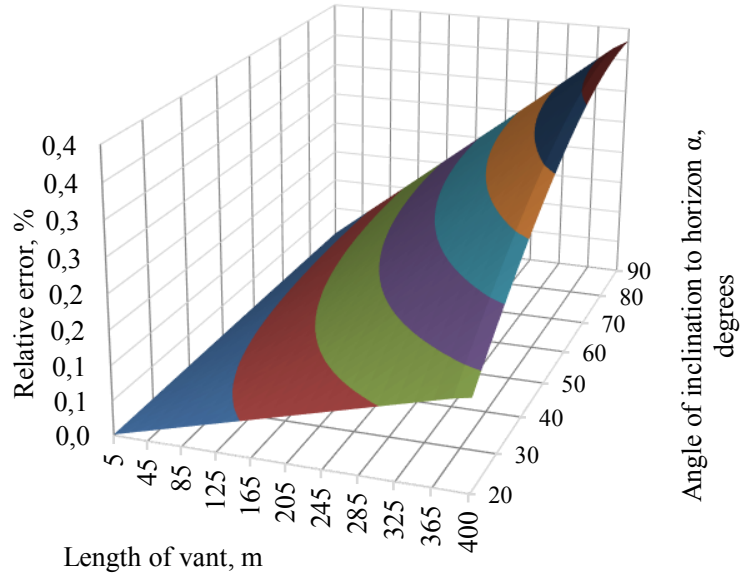

Fig. 4. The surface of the relative error of the tension force of the cable stay without taking into account the effect of its angle of inclination to the horizon.

According to the results of theoretical studies, when the values of the parameters in the selected ranges change, the maximum relative error $\delta_{\alpha}$ does not exceed $4 \%$. But usually the ratio of the parameters of the cable stays is that the relative error of the tension force of the cable stay without taking into account the influence of the angle of inclination of the cable stay to the horizon is even less and does not exceed $1 \%$.

\subsection{Effect of bending stiffness of the cable stay on its tension force}

It is generally accepted to consider a cable stay as a flexible thread, since its length is much smaller than its transverse dimensions. However, it is indicated in [12] that in determining the frequencies of the transverse oscillations of the cable stay, it is necessary to take into account its bending stiffness. The frequency of the natural oscillations of the cable stay taking into account its bending stiffness can be determined by the following formula [12]: 


$$
v_{n}^{b}=\frac{n}{2 l} \sqrt{\frac{N}{m}+\frac{(n \pi)^{2} E I}{l^{2} m}}
$$

where $I$ - moment of inertia of a cable stay, $\mathrm{m}^{4}$.

Taking (11) into account, we obtain:

$$
N_{w . b .}=N+N_{b}
$$

where $N_{b}=\frac{(\pi n)^{2} E I}{l^{2}}$ - force depending on the bending stiffness of the cable stay, N.

The surface of the relative error in the tension force of the cable stay $\delta_{b}$ (see formula (7)) without taking into account its bending stiffness is shown in Figure 5. When plotting the graph in Figure 4, the tension force of the cable stay is conventionally taken to be 935 $\mathrm{kN}$.

The error increases with the increase in the cross section of the cable stay and the decrease in its length (see Figure 5). The maximum relative error $\delta_{b}$ can exceed $100 \%$. But, as a rule, the ratio of the parameters of the cables is that the relative error of the force of the cable-stayed element without taking into account the influence of its bending stiffness does not exceed $3 \%$.

It should be noted that in the bottom part of the cable stay, protective (vandal proof) structures are usually installed (for example, metal galvanized pipes are installed on the cable-stayed elements of the Bugrinsky Bridge). Therefore, to the short cable stays, it is necessary to additionally take into account the influence of the stiffness of the protective structures when determining the forces in the cable stays by the frequencies of the natural transverse vibrations.

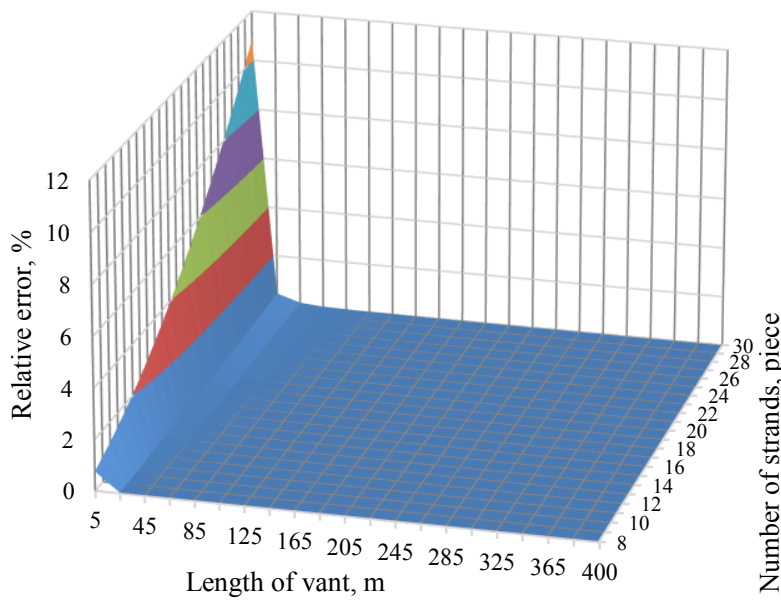

Fig. 5. The surface of the relative error of the tension force of the cable stay without taking into account the effect of its bending stiffness.

\subsection{Effect of temperature change on the tension force of the cable stay}

The change in the tension forces of the cable stays is possible with an uneven heating of the structural elements of the span structure as a whole. With a smooth temperature change and the design work of the bearing parts of the bridges, it can be assumed that there will be no additional forces, since the coefficients of linear expansion of the bridge elements' materials are close to each other. However, with a sharp change in temperature (usually due to the effect of wind and sun), the cable stays heat up and cool down faster because they 
have less heat capacity than the rest of the bridge elements, they will have additional forces from the temperature difference. According to our own research and the results given in [2], the magnitude of the abrupt temperature change can reach up to $15{ }^{\circ} \mathrm{C}$. The effect of the change in the force in the cable stay $N_{t}$ with the temperature changes $\Delta T$ and with allowance for Hooke's law can be obtained from the formula [10]:

$$
N_{t}=\alpha_{t} A E \Delta T
$$

where $\alpha_{t}$ - coefficient of linear expansion of metal, $\frac{1}{\Delta T^{0} \mathrm{C}}$;

$\Delta T$ - temperature change, ${ }^{0} \mathrm{C}$.

Note that the change in the force in the cable stay $N_{t}$ does not depend on the length of the cable stay.

The force in the cable stay taking into account the temperature change can be determined by the formula:

$$
N_{\mathrm{t}}=N \pm N_{t}
$$

In Figure 6, the surface of the relative error of the tension force of the cable stay taking into account the effect of temperature change $\delta_{\mathrm{t}}$ is constructed (according to formula (7)). When plotting the graph in Figure 6, the tension force of the cable stay is conventionally taken to be $935 \mathrm{kN}$.

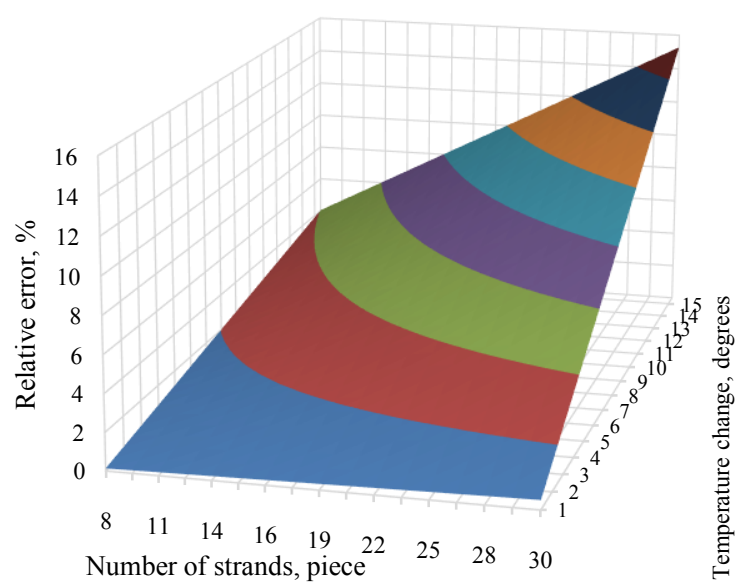

Fig. 6. The surface of the relative error of the tension force of the cable stay without taking into account the effect of temperature change.

The relative error in determining the tension force of the cable stay without taking into account the effect of temperature change may exceed $100 \%$. However, usually the ratio of the parameters of the cable stays is that the maximum relative error of the tension forces of the cable stays without taking into account the effect of the temperature change does not exceed $20 \%$.

\section{Conclusions on theoretical studies}

The relative error of the tension force of the cable stay without taking into account its sagging, angle of inclination, and bending stiffness is no more than $1 \ldots 3 \%$, therefore, in determining the tension forces, these force components can be neglected. When determining the tension force of the cable stay, it is necessary to take into account the length of the anchors participating in joint oscillations with the cable stay. Significant 
relative errors (up to 20\%) in determining the forces in the cable stays can be caused by temperature differences from the uneven heating of the elements, so it is recommended to carry out measurements in cloudy windless weather, and also to record the temperature of the cable stay and the surrounding structures.

\section{Results of experimental studies}

\subsection{Specifics of determining the forces in the cable stays by the frequencies of natural oscillations}

When measuring the natural oscillations of the cable stays, a large number of forms can appear (for example, when determining the frequency of natural oscillations in the cable stays on a road bridge across the Ob River in the city of Surgut, up to 20 forms of oscillations were observed [3, 13], at the Bugrinsky Bridge across the Ob River in Novosibirsk - up to 15 forms. [10] This causes the shortcomings of most methods for determining frequencies - to record all forms of oscillations, it is necessary to install a large number of accelerometer sensors along the length of the cable stay and use special equipment (for example, portable telescopic tower) [1]; special load cells for determining the tension forces of the cable stays are quite expensive, and, besides this, the rearrangement of the equipment is a complex and time-consuming process. However, all these shortcomings can be mitigated if a sensitive sensor (for example, vibration sensor accelerometer "Tensor MS") is set in one of the fixing points, where all forms of oscillations of the cable stay occur and are recorded.

The cross-sectional shapes of the cable stay may vary, which may affect the determination of the forces in the cable stays by the frequencies of natural oscillations. The process of allocating the natural multiple frequencies of oscillations of the cable stays consisting of strands is more complicated than the ones with a solid cross section. It is difficult to set vibration sensors on each strand because of their close location and a small cross-section of the strands themselves in the process of tension, and is impossible in the process of operation because of the presence of an anticorrosive casing. However, despite this, the necessary measurements can be carried out. The vibration sensor in such cable systems can be installed on the anchor device [14], but the process of processing and selecting multiple frequencies will be more difficult. When processing about 1000 records of oscillations of the cable stays of different bridges, the multiple frequencies are not explicitly distinguished and special signal processing is needed in $15 \%$ of cases $[15,18]$. For example, Figure 7 shows the frequency spectrum of oscillations of one of the bridge's cable stays across the river Irtysh on the bypass of Pavlodar (Republic of Kazakhstan), which clearly shows the presence of many peaks at close frequencies. One of the reasons for this phenomenon can be the difference in the tension of individual strands in the cable stay. On the one hand, this makes processing difficult, but, on the other hand, it can be an additional diagnosed parameter characterizing the quality of strands' tension. 




Fig. 7. Frequency spectrum of oscillations of a cable stay assembled from strands.

Another special feature of determining the force in the cable stays by frequencies of natural oscillations is the choice of a plane for measurements - vertical or horizontal, transverse to the longitudinal axis of the bridge. Numerous experiments and analysis of the obtained data have shown that it is sufficient to record only the frequencies of the transverse oscillations of the cable stay. The choice of the horizontal transverse plane is also caused by the fact that vertical oscillations of the cable-stayed girder occur in the vertical plane. As a rule, the spectrum of oscillation frequencies of the span structure of bridges with cable-stayed elements is quite wide, which can introduce additional peaks in the frequency spectrum when measuring the oscillations of the cable stays and increase the errors in determining the forces.

In the process of operation, the results of measurements can also be affected by the passage of a heavy reversing load and the randomness of the traffic flow, creating noise interference when recording vibrations. On the basis of the analysis of the results of force measurements in cable-stayed elements, the minimum required time for recording the dynamic parameters (recording length) - $90 \mathrm{sec}$, is obtained experimentally, which provides the possibility of separating the natural frequencies of oscillations. In some cases, the recording time can be increased. It should be noted that in addition to the frequencies of the vertical oscillations of the span structure, forcing frequencies can also present in the frequency spectrum caused by wind pulsations. In order to minimize their influence, it is necessary to carry out measurements in calm weather, for example, on the Ugra Bridge it is recommended to measure the frequencies of the natural oscillations of the cable stays at a wind speed of no more than $5-7 \mathrm{~m} / \mathrm{s}$ [6].

It should be noted that when oscillations of two neighboring cable stays are excited, their frequencies will be recorded in both elements, overlapping each other. This fact was confirmed by a simple experiment carried out by the authors. During the construction of the Bugrinsky Bridge, measurements of the frequencies of neighboring cable-stayed suspensions were made, exciting oscillations in them with small impulse forces. The measurements were carried out at the time of the absence of heavy working equipment on the bridge. The recording of the oscillations was carried out for 5 minutes. The first 2.5 minutes with "small" pulses acted on one cable-stayed suspension, in the second half of the recording time - on the second. In the frequency spectrum of cable-stayed suspension, which was in a quiet state (without the application of impulse actions), there were natural frequencies of oscillations of the neighboring suspension unbalanced and performing free oscillations. 
A large number of cable-stayed elements themselves and their natural oscillation frequencies require considerable time in the processing of measurement results in the nonautomatic mode. To automate these processes, specialized software "Vant" ("Cable stay") was developed [20]. The "Vant" program implements specially designed filters for the forced oscillation frequencies of the cable stays, algorithms for determining the frequencies of the natural oscillations of the cable stay and calculating the characteristics of damping and dissipation of energy, etc. It is also possible to determine the force for one cable stay and for all the cable stays of the same structure.

\subsection{Influence of the fixing structures of the cable stays}

In order to determine the influence of the conditions of fixing the cable stays on the frequencies of their natural oscillations, the work of the anchors installed on the span structure of the Bugrinsky Bridge was analyzed. For the study, 42 anchors (out of a total of 156 pcs.) were selected. At the measurements, six vibration sensors - accelerometers "Tenzor MS" were used. Vibration sensors were oriented in a horizontal plane across the longitudinal axis of the bridge and installed at characteristic points (places of variation of bending stiffness) along the length of the anchor. The recording time on each anchor was about three minutes. Figure 8 shows a diagram of the installation of vibration sensors along the length of the anchor.

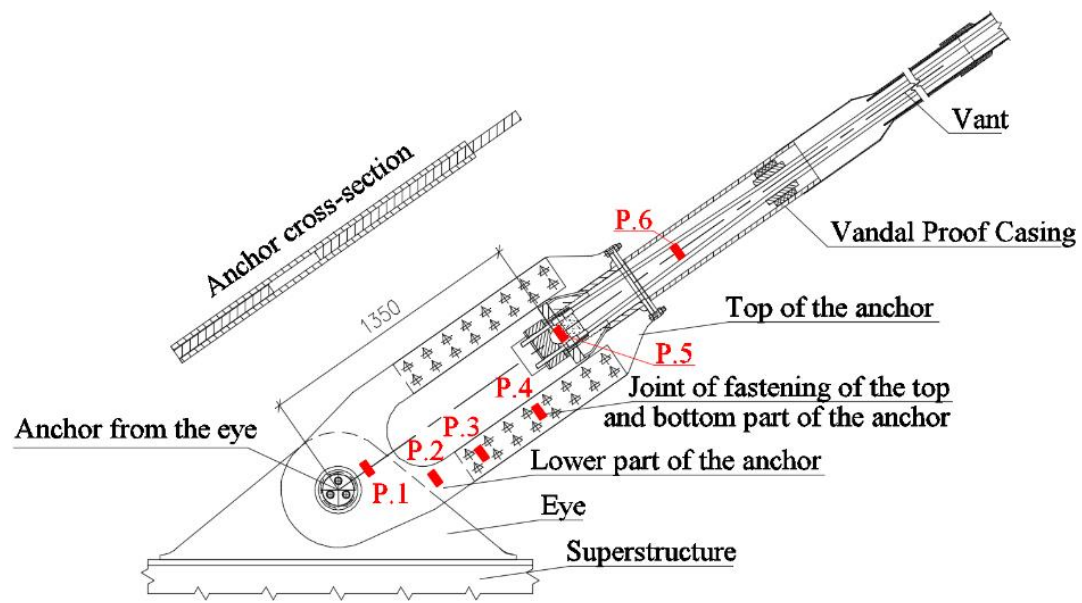

Fig. 8. Scheme of installation of vibration sensors along the length of the anchor.

According to the processing of the vibration records, the surface and the graph (based on averaged values) of the relative amplitude of the oscillations of the sensor mounting points at the same time were constructed (point № 5 (see Figure 8) was chosen as a point of "relativity", in which sensors were installed mandatory for all measurements in all cablestayed suspensions). The surface and the graph plotted by the averaged values of the relative amplitude of the oscillations of the anchor points are shown in Figures 9 and 10. 




Fig. 9. Surface of change in the relative amplitude of the anchor points' oscillations.

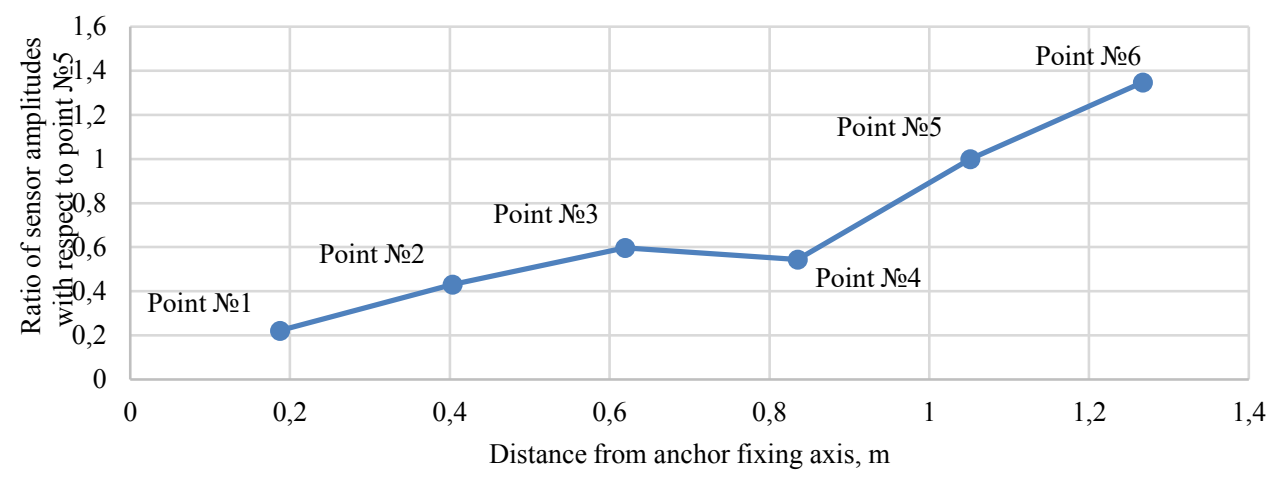

Fig. 10. A graph plotted by the averaged values of the relative amplitude of the anchor points' oscillations.

It can be seen from Figure 9 that the character of the anchor movements does not depend on the length of the cable stay, and Figure 10 clearly shows that the anchor oscillates with the cable stay. As a result of the calculation of the natural oscillation frequencies of the cable stay, it was concluded from the formula (3) that the results closest to the measured values can be obtained by taking into account the length of the anchor in the total length of the oscillating thread. In addition, it was found that in the total mass of the vibrational system, it is necessary to take into account the masses of the external anticorrosive casing and the vandal proof casing, distributed along the length of the cable stay.

With sufficient accuracy for practical use, the force in the cable stay can be determined as the average value of the forces determined by the first three frequencies of the natural oscillations of the cable stay. The formula for determining the force in the cable stay $N_{\text {vant }}$ is as follows: 


$$
N_{\text {vant }}=\frac{\sum_{1}^{3} N_{n}}{3},
$$

where $N_{n}=\frac{\left(m+\frac{M}{L_{p}}\right) \cdot\left(2 L_{p} f_{n}\right)^{2}}{n^{2}}-$ force in the cable-stayed element, N, $M$ - mass of the vandal proof casing, $\mathrm{kg} ; f_{n}$ - the $n$-th actual natural oscillation frequency of the cable-stayed element, Hz; $L_{p}=l+L_{a n c}$ - the design length of the cable-stayed element, $\mathrm{m}$, equal to the sum of the length of the actual cable-stayed element $l$ and the design length of the anchor $L_{\text {anc }}$.

The forces in the cable stays according to the formula (15) were verified by comparing the forces measured by the frequencies of the natural oscillations of the cable stay with the values of forces obtained by the specialists of the VSL company according to the readings of the manometers on the jacks under tension of the cables of the Bugrinsky Bridge [10]. The error between the forces received by specialists of the VSL and authors, as a rule, does not exceed 5\% [10].

\subsection{Effect of temperature change on the natural oscillation frequencies of the cable stay}

The effect of the temperature change on the natural oscillation frequencies of the cable stay was studied by means of long measurements on the longest cable-stayed suspension of the Bugrinsky Bridge. The vibration sensor was installed on the anchor to record the natural oscillation frequencies. The recording length for each measurement was about three minutes. The temperature of the cable-stayed suspension and tightening (span) was measured by means of a Raytek Mini Temp MT4 ${ }^{T M}$ Portable IR Thermometer intended for remote temperature measurement by a non-contact method (with a temperature measurement accuracy of $0.1{ }^{\circ} \mathrm{C}$ ). The data was collected every $35 \ldots 60$ minutes from 6:46:32 to 12:24:53, on August 24, 2014. During this time, the temperature of the structures increased by an average of $14{ }^{\circ} \mathrm{C}$. The maximum temperature difference between the elements at the moments of measurement reached $5{ }^{\circ} \mathrm{C}$. Table 1 shows the results of the study of the effect of temperature change on the forces in the cable stay, and in Figure 11 the graph of the change in the force in the cable-stayed suspension from the temperature.

Table 1. Results of the study of the effect of temperature change on the forces in the cable stay.

\begin{tabular}{|c|c|c|c|c|c|c|c|c|c|}
\hline \multicolumn{2}{|c|}{ Record start time } & $\begin{array}{l}6: 46: \\
32\end{array}$ & $\begin{array}{l}7: 33: \\
13 \\
\end{array}$ & $\begin{array}{l}8: 29: \\
07 \\
\end{array}$ & $\begin{array}{l}9: 12: \\
09\end{array}$ & $\begin{array}{l}\text { 10:02: } \\
57\end{array}$ & $\begin{array}{l}10: 40: \\
43\end{array}$ & $\begin{array}{l}\text { 11:30: } \\
07\end{array}$ & $\begin{array}{l}\text { 12:24: } \\
53\end{array}$ \\
\hline \multirow{3}{*}{ 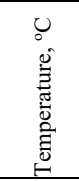 } & Cable-stayed suspension * & 14.4 & 15.4 & 16.6 & 17.0 & 19.4 & 21.0 & 24.4 & 26.9 \\
\hline & Tie rod* & 14.4 & 16.6 & 17.5 & 16.0 & 19.4 & 22.3 & 27.5 & 30.0 \\
\hline & Temperature change in suspension & 0.0 & 1.0 & 2.2 & 2.6 & 5.0 & 6.6 & 10.0 & 12.5 \\
\hline \multirow{4}{*}{ 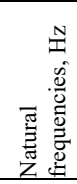 } & $f_{2} / 2$ & 0.732 & 0.732 & 0.732 & 0.732 & 0.732 & 0.732 & 0.732 & 0.732 \\
\hline & $f_{3} / 3$ & 0.749 & 0.749 & 0.716 & 0.716 & 0.749 & 0.749 & 0.749 & 0.716 \\
\hline & Average frequency & 0.754 & 0.754 & 0.743 & 0.743 & 0.754 & 0.754 & 0.754 & 0.743 \\
\hline & Change in frequency $* *$ & 0.000 & 0.000 & $\overline{0} .011$ & $\begin{array}{l}- \\
0.011\end{array}$ & 0.000 & 0.000 & 0.000 & -0.011 \\
\hline \multirow{2}{*}{$\begin{array}{l}Z \\
\text { Z } \\
\dot{0} \\
\dot{0} \\
0\end{array}$} & Force in cable stay & 388 & 388 & 377 & 377 & 388 & 388 & 388 & 377 \\
\hline & Change in force $* *$ & 0 & 0 & -11 & -11 & 0 & 0 & 0 & -11 \\
\hline
\end{tabular}

Note: * - The temperature of the suspension and tie rod was measured at several points of the corresponding structure. The table shows the average temperature for each element;

** - The change in frequency and force is given relative to the values recorded at 6:46:32. 


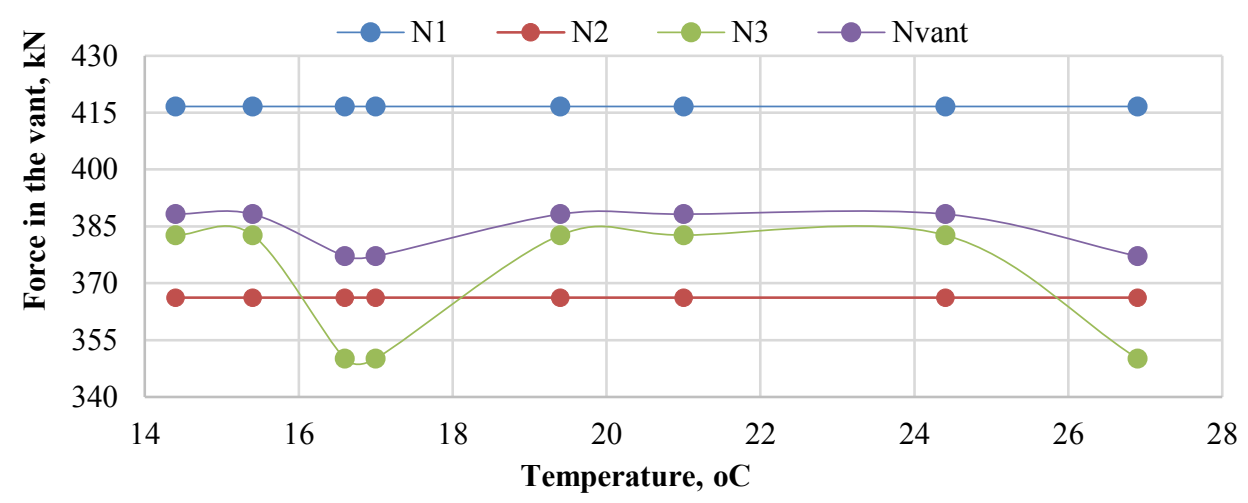

Fig. 11. The graph of the change in the forces in the cable-stayed suspension of the Bugrinsky Bridge from the temperature.

As can be seen from Table 1 and the graph in Figure 11, the force in the cable stay has not changed. In the cable-stayed suspension under consideration, the design value of the change in the force in the cable stay when the temperature is changed by $1{ }^{\circ} \mathrm{C}$, calculated from the formula (13), is $5 \mathrm{kN}$ ( $1.3 \%$ of the tension force).

Accordingly, if the bearing members were not working correctly and the temperature of the structure increased by $14{ }^{\circ} \mathrm{C}$, the force in the cable stay could be reduced by $70 \mathrm{kN}$, but the bearing members worked normally and the tensile force of the cable-stayed suspension did not decrease. Insignificant changes of the forces in the cable-stayed suspension recorded at 8:29:07, 9:12:09, and 12:24:53 (see Table 1) probably occurred because of rapid temperature changes.

\subsection{Influence of energy dissipation of oscillations on frequencies of natural oscillations of the cable stays}

Studies of energy dissipation of oscillations were carried out at the Bugrinsky, Ugra, and Vinogradovsky bridges. When analyzing the energy dissipation of oscillations, the property of the multiplicity of the natural frequencies of the transverse oscillations of the tensioned thread was used - the value of the first natural oscillation frequency of the cable stay was compared with the subsequent ones divided by their own order number.

Automation of the process of separation of natural oscillation frequencies [20] made it possible to accelerate the processing of measurement results and to estimate the energy dissipation. Note that the values obtained should decrease with increasing sequence number, since the higher the frequency, the greater the energy dissipation.

However, at close values of the frequencies of natural oscillations of cable-stayed suspensions with background oscillation frequencies (oscillation frequency of the span structure, external periodic effects, etc.), the frequency peaks can shift to background values, which can complicate determination of the true natural frequency.

Table 2 summarizes the data on the change in the natural oscillation frequencies of the cable stays, and in Figure 12, the graphs of variation of the natural frequencies of the oscillations of the cable stays reduced to the first frequency $(n=1)$ are given depending on the order number of the oscillations $(n)$. 
Table 2. Data on the variation of the natural oscillation frequencies of the cable stays.

\begin{tabular}{|c|c|c|c|c|c|c|c|c|c|}
\hline \multirow[b]{4}{*}{ Name of the bridge } & \multicolumn{9}{|c|}{ Changing the frequencies of natural oscillations, $\%$} \\
\hline & \multicolumn{7}{|l|}{$\mathrm{n}$} & \multirow{3}{*}{$\begin{array}{l}\text { Minimum } \\
\text { value } \\
\text { among all } \\
\text { the } \\
\text { bridge } \\
\text { cable } \\
\text { stays }\end{array}$} & \multirow{3}{*}{$\begin{array}{l}\text { Maximum } \\
\text { value } \\
\text { among al } \\
\text { the bridge } \\
\text { cable } \\
\text { stays }\end{array}$} \\
\hline & 2 & 3 & 4 & 5 & 6 & 7 & 8 & & \\
\hline & \multicolumn{7}{|c|}{ Average value calculated for all the bridge cable stays } & & \\
\hline Bugrinsky Bridge & -2.0 & -2.8 & -4.0 & -4.7 & -4.5 & -5.3 & -5.4 & -37.4 & 30.1 \\
\hline Ugra Bridge & 0.1 & 0.5 & 0.4 & 0.4 & 0.4 & 0.3 & 0.3 & -19.8 & 12.9 \\
\hline Vinogradovsky Bridge & -0.7 & -0.6 & -0.2 & 0.3 & 0.8 & 1.3 & 2.0 & -9.1 & 15.0 \\
\hline Average value & -0.9 & -1.0 & -1.3 & -1.4 & -1.2 & -1.3 & -1.1 & -22.0 & 19.3 \\
\hline
\end{tabular}

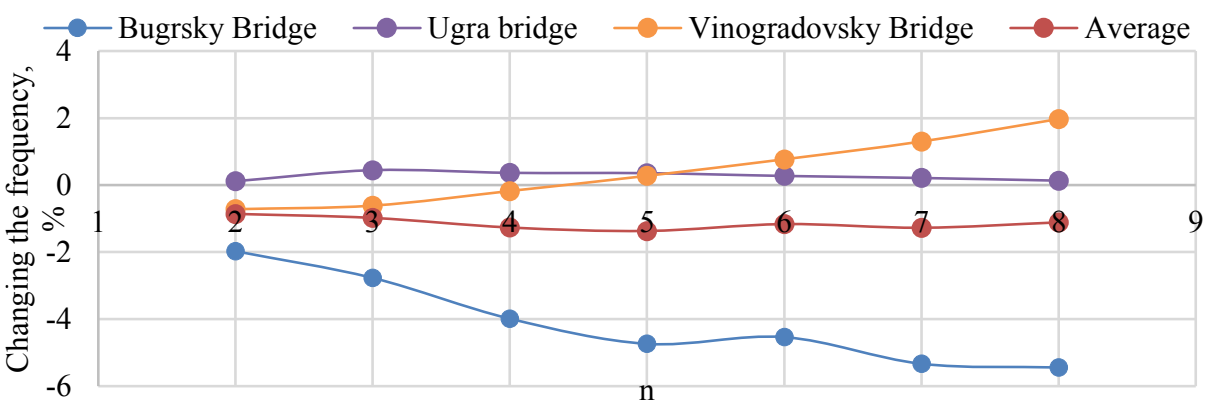

Fig. 12. Graphs of changes in the natural oscillation frequencies of the cable stays.

The maximum decrease in the frequencies of natural oscillations is recorded on the cable stays of the Bugrinsky Bridge, which is due to the presence of increased resistance because of the design of the cable stays themselves (strands in a protective anticorrosive casing). On the cable stays of the Ugra Bridge, which is also noted in the studies [6], the phenomenon of energy dissipation makes insignificant changes in the natural oscillation frequencies of the cable stays (within one percent). On the Vinogradovsky Bridge, the phenomenon of energy dissipation is not recorded, but, on the contrary, there is an increase in the natural oscillation frequencies of the cable stays divided by their order number (see Figure 12). This is due to the fact that the cable stays on the pylon are rigidly fixed in a single "saddle", which causes a constant inflow of vibrational energy from one cable stay to another.

It should be noted that the change of the first three frequencies divided by their order number does not exceed three percent on all bridges. Therefore, taking into account the obtained study results, it is recommended to determine the forces in the cable stay by the first three natural oscillation frequencies (see formula (15)).

\section{Conclusions on experimental studies}

The results of experimental studies indicate a complex dynamic work of cable-stayed elements in the bridge structures. When determining the forces in the cable stays, it is necessary to take into account the structural features of the cable-stayed system, to record the temperature difference between the cable stay and the surrounding structures, and to carry out measurements in a windless cloudy weather and, if necessary, repeatedly control the force in a short time after change in the weather conditions. The work of the fixing structures participating in the oscillatory process must be taken into account by adding the 
length of the anchor to the length of the cable stay. When calculating the force in the cable stay, one must take into account the mass per unit length of the external anticorrosion casing and the mass of the vandal proof casing distributed over the entire length of the cable stay. The force in the cable stay is recommended to be determined from the first three frequencies of natural oscillations in order to minimize the errors associated with the change in the frequencies of natural oscillations with increasing their order number. To ensure the reliability of the results obtained, the measurement equipment must be certified and verified. Data processing is best done using specialized software to eliminate subjectivity in estimating the frequency and speeding up the processing of measurement results.

\section{General conclusions}

The conducted studies confirm the possibility and expediency of the organization of the monitoring system of the stress-strain state of cable-stayed bridges by changing the natural oscillation frequencies of the cable stays. Regularly conducting periodic automated measurements in the monitoring system will increase the reliability of determining the assessment of the technical condition, promptly detect the significant emergent defects affecting the load capacity and rapidly take measures to eliminate them, which will extend the time and increase the safety of the bridge operation.

In the future, it is planned to implement the developed method for other bridge structures with cable-stayed elements, and also to study the damping characteristics of the oscillations (damping coefficient and logarithmic decrement).

\section{References}

1. A. Ladysz, R. Casas, R. Joan, Structural health monitoring and life-cycle costing of structures. Application to cable-stayed bridges (Universitat Politècnica de Catalunya, 2009)

2. CIP recommendations on cable stays (Setra, France, 2002)

3. V.F. Solokhin, The road bridge across the Ob river near the city of Surgut: features of design and construction: textbook (Sarat. state. tech. University, Saratov, 2002)

4. S.N. Dyadkin, Justification, cantilever construction technology, and monitoring of the cable-stayed span structures taking into account climatic factors (for example, a bridge across the Ob river near the city of Surgut): thesis of Cand. Sc. (Saratov, 2004)

5. P.A. Sychev, Methods for assessing the stress-strain state of cable stays and substantiating the technical requirements for cable-stayed systems of bridges: thesis of Cand. Sc. (Moscow, 2006)

6. A.P. Pedora, V.P. Fayantsev, Report "Measurement of tension forces of the cable stays" (OOO SC “TsAGI-TEST”, 2004)

7. G.A. Belov, V.A. Nanosov, A.P. Pedora, A. Smotrov, V.P. Fayantsev, K.S. Strelkov, Determination of the tension forces of the cable stays of the channel span structure from the test loads: the scientific and technical report (OOO SC "TsAGI-TEST", 2000)

8. M.I. Khazanov, Analysis of the stress-strain state of structures using a computer measuring system: thesis of Cand. Sc. (Moscow, 2007)

9. A.A. Petropavlovskii, Cable-stayed bridges (Transport, Moscow, 1985)

10. I.V. Chaplin, A.N. Yashnov, Engineer's Handbook 10, 56-63 (2014) 
11. D.J. Farquhar, ICE Manual of Bridge Engineering, Institution of Civil Engineers, 357381 (2008)

12. M.M. Korneev, Steel bridges: theoretical and practical manual on designing (Kiev, 2003)

13. I.V. Chaplin, P.Yu. Kuzmenkov, Materials of the conference "Innovative factors of transport development. Theory and practice: intern. scientific-practical conf .: theses of reports" (2017)

14. As-built surveys when monitoring the stress-strain state of bridge structures across the river Irtysh in the structure of object "Construction (reconstruction) of the road "Bypass of Pavlodar", 3d section, Bridge crossing over the Irtysh River with regulatory facilities" (SSTU, Novosibirsk, 2016)

15. I.I. Snezhkov, I.V. Chaplin, A.N. Yashnov, Journal "SSTU Bulletin" 4, 18-25 (2017)

16. A.N. Yashnov, I.V. Chaplin, I.I. Snezhkov, Patent of the Russian Federation №2613484. IPC G 01 L 1/10, G01L 5/04, Method for determining the tension forces of the cable-stayed element of the bridge

17. A.N. Yashnov, I.V. Chaplin, The Bulletin of the RSTU 4, 135-141 (2017)

18. I.I. Snezhkov, Track and track facilities 9, 25 (2007)

19. I.I. Snezhkov, P.Yu. Kuzmenkov, Instruments and methods of measurement, quality control and diagnostics in industry and transport, 101-105 (2013)

20. A.N. Yashnov, I.I. Snezhkov, I.V. Chaplin, Yu.S. Ivanov, Certif. 2018611633 Russian Federation. Certificate of state registration of the computer program Vant 